\title{
Heart rate responses of the hooded rat to low intensity white noise onset and offset
}

\author{
I. R. INGLIS* \\ University of Bristol, Bristol, BS8 $1 \mathrm{HH}$, England
}

\begin{abstract}
The heart-rate responses of male hooded rats to the novel offsets or onsets of a low-intensity white noise were measured. Basal resistance levels of the Ss were monitored concurrently with the heart rate in order to assess whether the intensity of stimulus used was stressful to the animals. No change in the basal resistance levels was found over the trial, suggesting that the noise level was not stressful. A brief deceleration of heart rate was found as a response to both types of test stimulus. These findings are consistent with the view that the heart-rate acceleration in response to a novel auditory stimulus reported by some earlier workers was probably produced by a defense reflex, which in turn was triggered by the higher intensity of stimulus used in these experiments.
\end{abstract}

A basic divergence of thought concerning the cardiac components of the orienting reflex stems from the work of Sokolov (1960) and Lacey and Lacey (1958). Sokolov suggested that the increased responsiveness to environmental stimulation occurring during the orienting reflex is accompanied by heart-rate acceleration. Lacey and Lacey (1958), however, argued that increased heart rate leads, via innervation from aortic baroreceptors, to inhibition of cortical activity. This inhibition was in its turn believed to produce a reduction in the animal's sensitivity to environmental stimulation. Therefore, if this argument is true, if an external event requires attention, as during an orienting response, it would be expected that cardiac deceleration should be present if indeed there is any cardiac change at all.

One of the basic properties of the orienting response was held to be its independence of the direction of stimulus change. Experiments using simple, monomodality stimuli (usually auditory) have, however, shown that heart-rate responses to such stimuli can be qualitatively different in this respect. For example, Soltysik et al (1961) and Shearn (1967) both report that white noise onset produces heart-rate acceleration, while white noise offset produces heart-rate deceleration. Other workers investigating the effects of noise onset, e.g., Fehr and Stearn (1965), or noise offset, e.g., Robinson and Gantt (1947), have obtained results that agree in the main with the above pattern. If the test stimuli used are repetitive, then diphasic patterns of heart-rate change may result, e.g., Lang and Hnatiow (1962).

Graham and Clifton (1966) have suggested that the intensity of the auditory stimuli used in previous experimentation was such as to elicit a defense reflex which could have confounded any cardiac change [e.g., Shearn (1967) used a test intensity of $70 \mathrm{~dB}$ ]. If Lacey and Lacey are correct, then the cardiac response to

*I would like to thank N. Freeman, Psychology Department, Bristol University, for his critical reading of this manuscript, and $R$. Wootton, Zoology Department, University College of Wales, Aberystwyth, for statistical advice. strong environmental stimuli should be heart-rate acceleration, as this leads to decreased cortical sensitivity to environmental stimuli. The final observed direction of cardiac change may therefore depend upon the relative predominance of one of these reflexes over the other, both being elicited by the same strong "novel" auditory stimulus. It should be noted, however, that the final stimulus intensity may not be the only factor controlling any cardiac change, for Flesher (1965) has reported that behavioral startle in rats is a function of stimulus intensity rise time rather than of the final intensity level.

In the present experiment, the cardiac responses of rats resulting from experiencing either a low-intensity white noise offset stimulus or a low-intensity white noise onset stimulus were compared. The test intensity was $40 \mathrm{~dB}$. As a means of checking that this low-intensity stimulus did indeed produce no defense reflex, the basal resistance levels of the Ss were monitored concurrently with the heart rate. Workers have shown (e.g., Kaplin, 1963; Blizzard, 1968) that the basal resistance levels of rats fall when animals are subjected to what are normally termed aversive stimuli. Further, a pilot study had already shown that noise intensities of the order of those used in the above experiments produced a drop in the basal resistance levels of rats of at least 20\% (Inglis, unpublished results). If, therefore, any significant drop in the basal resistance levels of the Ss was found during the present experiment, this would suggest that stimuli of only $40 \mathrm{~dB}$ can produce defense reflex complications. If, however, no drop was found, then this would lend additional support for the assumption that only orienting responses were elicited by the test stimulus.

\section{METHODS}

\section{Subjects}

The Ss were 10 experimentally naive 4-month-old male Lister hooded rats. Three weeks before the initial trial of the experiment, the animals were operated upon and heart-rate electrodes inplanted. The Ss were narcotized by an IP injection of Nembutal. Two small loops of stainless steel wire $0.022 \mathrm{~mm}$ in diam) were inplanted under the skin in the midline of the 
back of the animal, one near the shoulder blades and the other above the base of the tail. Microminiature connectors were crimped to the protruding ends of these loops. The animals were housed singly within polythene-walled cages $(0.5 \times 0.38$ x $0.20 \mathrm{~m})$.

\section{Apparatus}

The heart rates and basal resistance levels of the rats were recorded upon a Mingograph pen recorder. All electrical leads were fitted with E.M.T.17 main suppressors. Deaf-aid cord was used to connect the animal's electrodes to a swivel. The swivel allowed the $S$ reasonably unrestricted freedom of movement within the experimental chamber $(0.25 \times 0.25 \times 0.45 \mathrm{~m})$ without risk of it entangling the electrode leads. The experimental chamber was placed within an electrically screened chamber $(0.56 \times 0.56 \times 0.38 \mathrm{~m})$. A loudspeaker was built into the ceiling of the screened chamber and was connected via an amplifier to a white noise generator. The screened chamber containing the experimental chamber was placed inside a large soundproof testing room, all recording apparatus being housed in an adjacent room.

The method of Kaplin and Kaplin (1962) was used to monitor the basal resistance level. Adjacent parallel bars forming the floor of the experimental chamber were given opposite polarities such that when the animal short-circuited these bars a subthreshold current (approximately 5 microA) flowed through its body. A Wheatstone bridge circuit was then used to assess any resistance changes.

The S was placed within the experimental chamber, and the bridge circuit was adjusted in order to center the pen recorder trace. This bridge calibration was then kept constant until completion of the trial. After the trial, the $S$ was removed from the apparatus and known resistances used to calibrate the pen recording. An additional circuit periodically reversed the polarity of the bars throughout the trial period in order to minimize errors through polarization.

\section{Procedure}

The animals were randomly assigned to two experimental groups. Pretraining consisted of attaching the heart-rate leads to a $S$ while the latter was held in a small plastic transport cage. The animals tended to defecate when held and the heart-rate leads were initially attached; holding them in a small transport cage therefore minimized the chance of fecal boli being deposited in the actual apparatus. The S, complete with attached heart-rate leads, was then placed in the experimental chamber for $1 \mathrm{~h}$. This procedure was repeated on 4 consecutive days. Ss of Group 2 had white noise of $40 \mathrm{~dB}$ broadcast continuously to them for the whole time they were in the experimental chamber. On the 6 th day, the Ss were given the test trial. The animals were placed in the test chamber as before, and their heart-rate and basal resistance levels were continuously monitored. After $10 \mathrm{~min}$ Group 1 received a $5-\mathrm{sec}$ burst of $40-\mathrm{dB}$ white noise, while Group 2 experienced a 5-sec absence of 40-dB white noise. After the test stimuli, the heart rate and basal resistance levels were monitored for a further $5 \mathrm{~min}$.

\section{RESULTS}

Sometimes the Ss would fail to make good electrical contact with the bars of the floor of the experimental chamber, e.g., when sitting. In such cases, the resistance measured could be over $1,000 \mathrm{~K}$ ohms. The usual technique to circumvent this difficulty is to choose the lowest resistance level recorded (e.g., Blizzard, 1968; Kaplin, 1963). In this experiment, the lowest resistance reading, in every $1 \mathrm{~min}$ of the trial, which persisted for at least $5 \mathrm{sec}$ was used in further calculations. The other main sources of error in basal resistance level recording result from defecation and/or urination by the $\mathrm{S}$. Although both events occasionally occurred in the habituation trials, the majority of urination and defecation occurred in the small transport cage and no urination or defecation was observed in the test trial. The mean basal resistance levels for the prestimulus and poststimulus periods were calculated for each S. Correlated $\mathrm{T}$ tests failed to find any significant differences between the scores of the two periods for either of the two experimental groups (Table 1).

For every 1 -min period of the heart-rate trace, 10 1 -sec periods were picked at random, and the number of heart beats in each of these 1-sec periods was noted. A mean value for each minute of the trial was thus obtained. Any ambiguous portions of the trace created by a few gross movement artifacts were ignored (in these cases, the baseline of the trace would be off the paper for a brief period). T tests revealed no significant differences between the experimental groups over the whole trial in their heart rates.

Table 1

Mean Basal Resistance Levels in $\mathrm{K}$ Ohms

\begin{tabular}{clcc}
\hline $\begin{array}{c}\text { Experimental } \\
\text { Group }\end{array}$ & Period & Mean & SE \\
\hline \multirow{2}{*}{ Noise Off } & Prestimulus & 505 & 60.1 \\
& Poststimulus & 500 & 29.0 \\
\multirow{2}{*}{ Noise On } & Prestimulus & 473 & 29.1 \\
& Poststimulus & 516 & 34.0 \\
\hline
\end{tabular}

Figure 1 shows the more immediate heart-rate changes that occurred around the test stimulus presentation. For this analysis, the heart-rate trace was divided into four periods: a prestimulus period ending with the onset of the test stimulus, a stimulus period beginning with the test stimulus onset, and two poststimulus periods, the latter being two successive periods commencing with the offset of the test stimulus. Each of these periods for each animal's trace was composed of four consecutive 10-heart-beat sections. Each of the latter sections was used to compute a heart-rate score. Therefore, for every animal, there were obtained four clusters of heart-rate scores, each cluster corresponding to one of the periods and each consisting of four consecutive rate scores. An analysis of variance followed by a planned comparison of means (Edwards, 1960) was then performed on the data from the experimental groups. Unfortunately, the traces for three Ss (one in Group 1 and two in Group 2) were each obscured at one point by a large movement artifact (these did not seem to be a result of the test stimulus presentation, as they were all in different periods, including one in the prestimulus period). As a complete sequence of rates was needed for the analysis, the whole traces for these Ss were omitted from this calculation. For every $S$, a mean of the four rate scores was obtained in every period, and these figures were used to calculate the group means shown in Fig. 1. Upon 
Fig. 1. Mean heart rates around test stimulus presentation. Closed circles represent noise-off stimulus group. Open circles represent noise-on stimulus group. Bar length is standard error about the group mean. For further details, see text.

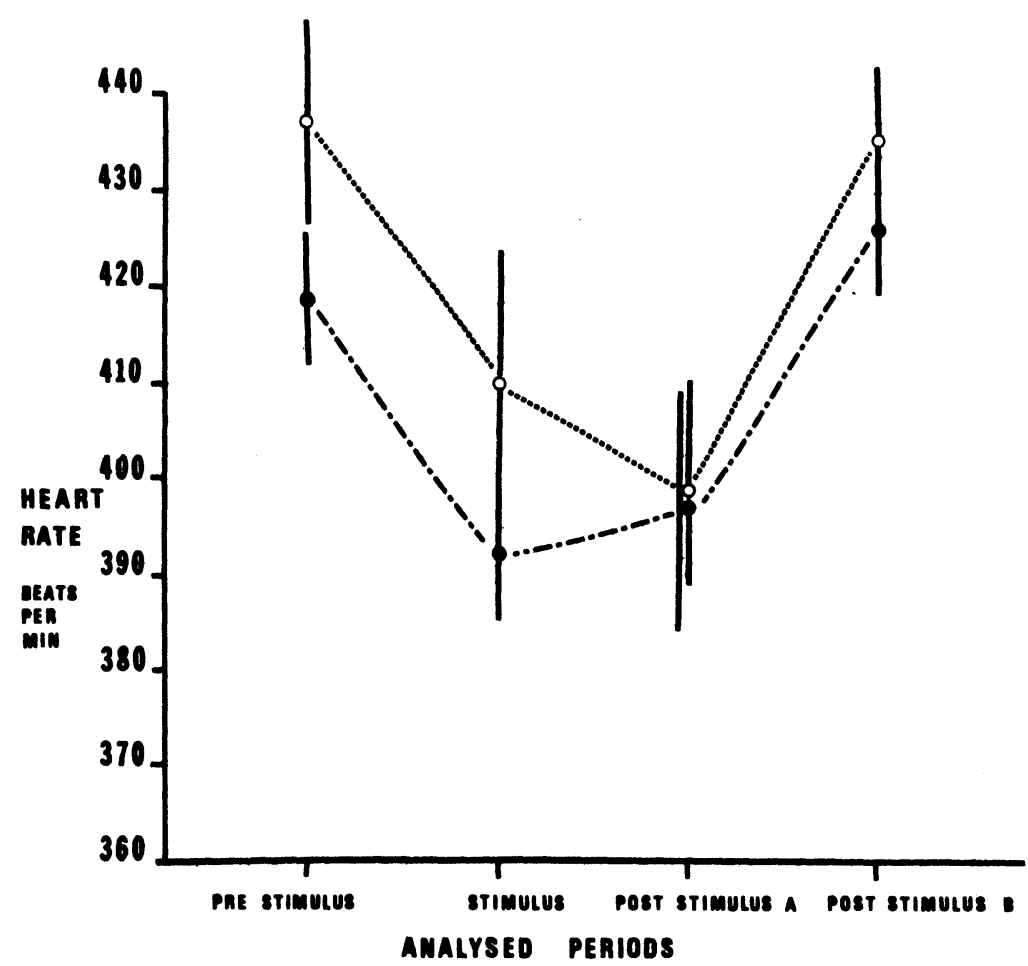

comparing the heart rates of the prestimulus and the stimulus periods, it was found that both experimental groups exhibited a decline in rate $(p<.01$ for both, one-tailed test). A similar comparison for the two poststimulus periods revealed that both groups showed a significant tendency to return to the prestimulus heart rate $(\mathrm{p}<.001$ for both, one-tailed test $)$.

\section{CONCLUSIONS}

The basal resistance level data give no indication that the intensity of white noise used in this experiment was such as to produce a marked defense reflex in the $S$. More credence can therefore be attached to the position that the cardiac changes revealed by this experiment are unfounded by defense reflex complications. It would seem, therefore, that the orienting reflex resulting from a brief, low-intensity auditory stimulus produces a cardiac deceleration around the onset of the stimulus regardless of whether the latter involved an increase or decrease in intensity. This finding is congruent with the original Lacey and Lacey (1958) position already discussed. The results also lend support to Graham and Clifton's argument (Graham \& Clifton, 1966) that the heart-rate increases reported by other authors may have resulted through a confounding defense reflex, cardiac change being elicited by the high intensity of the test stimuli used. It should also be remembered that, while these results do not agree with the above-mentioned position of Sokolov (1960), nevertheless, they are in agreement with one of his basic properties of the orienting reflex, namely that the latter is controlled solely by input stimulus discrepancy from expectation, within certain intensity limits, and not by the direction of that discrepancy away from expectation.

\section{REFERENCES}

Blizard, D. A. Autonomic and behavioural correlates of emotionality. PhD thesis, University College of Wales, Cardiff 1968 .

Edwards, A. C. Experimental design in psychological research. New York: Holt, Rinehart \& Winston, 1960.

Fehr, F. S., \& Stern, J. A. Heart rate conditioning in the rat. Journal of Psychosomatic Research, 1965, 8, 441-453.

Flesher, M. Adequate acoustic stimulus for startle reaction in the rat. Journal of Comparative \& Physiological Psychology, $1965,60,200-207$

Graham, F. K., \& Clifton, R. K. Heart rate change as a component of the orienting response. Psychological Bulletin, $1966,65,305-320$.

Kaplin, $R$. Rat basal resistance level under stress and non stress conditions. Journal of Comparative \& Physiological Psychology, 1963, 56, 775-777.

Kaplin, S., \& Kaplin, R. Skin resistance recording in the unrestrained rat. Science, 1962, 138, 1403-1404.

Lacey, J. I., \& Lacey, B. C. The relationship of resting autonomic activity to motor impulsivity. In The brain and human behavior (Proceedings of the Association for Research in Nervous and Mental Disease). Baltimore: Williams \& Wilkins, 1958. Pp, 144-209.

Lang, P. J., \& Hnatiow, M. Stimulus repetition and the heart rate response. Journal of Comparative \& Physiological Psychology, 1962, 55, 781-785.

Robinson, J., \& Gantt, W. H. The orienting reflex (questioning reaction): Cardiac, respiratory, salivary and motor components. Johns Hopkins Hospital Bulletin, 1947, 80, 231-253.

Shearn, D. Persistent and emergent cardiac responses elicited by simple stimuli. Conditioned R eflex, 1967, 2, 127-137.

Solty sik, S., Jaworska, K., Kowalski, M., \& Radom, S. Cardiac responses to simple acoustic stimuli in dogs. Acta Biologiae Experimentalis, 1961, 21, 235-252.

Sokolov, E. N. Neuronal models and the orienting reflex. In M. A. B. Brazier (Ed.), The central nervous system and behavior. New York: Josiah Macey Jr. Foundation, 1960. Pp. 187-276.

(Received for publication February 26, 1974; accepted March 26, 1974.) 